ISSN: $1130-3743$

\title{
CREATIVIDAD E INNOVACIÓN: UNA DESTREZA ADQUIRIBLE
}

\section{Creativity and innovation: an acquirable skill}

\section{Créativité et innovation: une habilité que l'on peut atteindre}

\author{
Petra María Pérez Alonso-Geta \\ Universidad de Valencia. Facultad de Filosofía y Ciencias de la Educación. \\ Departamento de Teoría de la Educación. Avda. Blasco Ibáñez, 30.46010 \\ Valencia.Correo-m: petra.m.perez@uv.es
}

Fecha de recepción: enero de 2009

Fecha de aceptación definitiva: abril de 2009

Biblid [(1130-3743) 21, 1, 2009, 179-198]

RESUMEN

Entendemos la creatividad como destreza adquirible, como un rasgo del que participan todos los seres humanos, aunque precise ser cultivada. La capacidad de ser creativo es una mezcla de conocimientos, actitudes y habilidades que se pueden conseguir mediante la práctica. Se trata de hacerse con nuevas ideas, saliendo de las rutas trazadas, por la experiencia para conseguir nuevos productos.

Desde la escuela es necesario estimular la creatividad para poder afrontar los retos que hoy se plantean, en los distintos ámbitos de la realidad, dando solución a los nuevos problemas. Educar para la creatividad es una estrategia de futuro.

Palabras clave: creatividad, innovación, educación, creencias, actitudes.

\section{SUMMARY}

This paper conceives creativity as an acquirable skill, as a trait shared by every human being. However, creativity must be developed: the ability of being creative is a blend of knowledge, attitude and skills that can only be learnt through practice. It 
is about having new ideas and departing from habitual pathways traced by everyday experience in order to obtain new outputs.

It is essential to stimulate creativity in school in order to be able to handle efficiently today's new challenges, which present themselves in different areas of our lives. Teaching creativity is therefore a strategy for the future.

Key words: creativity, innovation, education, knowledge, attitude.

\section{SOMMAIRE}

Nous définissons la créativité comme une habilité que l'on peut atteindre, comme un attribut auquel tous les êtres humains participent, même si elle a besoin d'être cultivée. La capacité d'être créatif est un mélange de connaissances, d'attitudes et d'habilités que l'on peut atteindre moyennant la pratique. Il s'agit de trouver des nouvelles idées, en sortant des chemins existants, à travers l'expérience afin d'obtenir de nouveaux produits.

Dès l'école il est nécessaire de stimuler la créativité pour pouvoir affronter les défis actuels, dans les différents domaines de la réalité en apportant des solutions aux nouveaux problèmes. Enseigner la créativité, c'est une stratégie pour l'avenir.

Mots clés: créativité, innovation, éducation, croyances, attitudes.

A don Ricardo Marín, gran artífice de la educación de la creatividad en España, con motivo de la declaración por la Unión Europea del 2009 como Año Europeo de la Creatividad y la Innovación.

El Año Europeo de la Creatividad y la Innovación tiene como primer referente la adopción en octubre del 2005 del "Plan D de democracia, diálogo y debate». Se inscribe dentro de los programas de la Comisión para promover una ciudadanía activa como apuesta de futuro. Se enmarca dentro de la Estrategia de Lisboa que tiene como objetivo hacer de Europa una sociedad basada en el conocimiento innovador y fundamentado en el potencial creativo.

Desde esta perspectiva, el Año Europeo 2009 responde a la necesidad de impulsar la capacidad de creatividad e innovación de la ciudadanía por motivos tanto sociales como económicos, ya que se considera que la creatividad y la innovación representan las fuentes del crecimiento, la inversión y los nuevos empleos. De ahí, que el papel que ha de desempeñar la creatividad, como recurso en el desarrollo social europeo, deba ir más allá de la educación y la cultura y ha de potenciar la capacidad humana de arbitrar soluciones creativas en los diferentes ámbitos de la realidad; dado que ello constituye una condición indispensable, para 
el desarrollo de personas activas que promuevan la innovación técnica, cultural y social $^{1}$.

\section{INTRODUCCIÓN}

El ser humano necesita innovar, ser creativo para poder vivir y progresar, el primer reto de su capacidad de innovación reside en su posibilidad de transformar en calidad de vida el conjunto de aptitudes, acciones y estrategias disponibles contando con el entorno.

La creatividad es una característica de la singularidad humana, que se define por ser propia del "ser que cavila", que piensa, que intuye la relación "causa-efecto", que es capaz de establecer objetivos y orden en sus prioridades. Para alcanzar sus fines, tiene que transitar, con frecuencia, por caminos nuevos, alborear lo que nunca ha sido: crear. Crea dando respuestas adaptadas a un medio ambiente cambiante; crea estableciendo escenarios y estrategias de futuro y crea diseñando, pintando, o solucionando un problema de la vida.

Para llevar a cabo la innovación no le es suficiente la experiencia acumulada. Innova cuando percibe una nueva necesidad y lo hace, revisando y combinando el conocimiento existente, en un proceso de intervención con el medio buscando la mejor situación. Cabe desde esta perspectiva interpretar la innovación como un proceso de aprendizaje cuyo recurso básico es el conocimiento, que es también a su vez su principal resultado. Se trata no obstante de un proceso complejo, que se alimenta tanto del conocimiento tácito como del conocimiento observable y especializado y que se puede ver afectado por una diversidad de factores. Algunos internos individuales (motivación, proactividad, etc.) y otros presentes en el entorno (educación, contexto social).

Torrance ${ }^{2}$, uno de los grandes teóricos de la creatividad, afirmaba ya en 1977 que hay muchas razones que nos obligan a pensar la educación del futuro en términos creativos. Los cambios en todo el universo son rápidos: los niños de hoy vivirán, cuando sean adultos, en un mundo profundamente diferente del actual; trabajarán en muchas ocasiones en profesiones que no existen todavía y que requerirán capacidades, destrezas, actitudes e información que aún no podemos imaginar. En esta nueva sociedad, la riqueza fundamental será el conocimiento, y estos cambios requerirán altos niveles de inventiva y creatividad. Sin entrar aquí

1. COM (2005) 494, d 13-10-2005. Decisión del Parlamento Europeo y del Consejo relativa al Año Europeo de la Creatividad e Innovacion 2009. COM 208-159 final 2008-2008/0064 (COD). http:// create2009.europa.eu.

2. Documento presentado en inglés, por el autor, en el Simposium Internacional de Creatividad, 22-17 de noviembre de 1976, en la Universidad Politécnica de Valencia, y traducido por Ricardo Marín Ibáñez. 
en delimitar los conceptos de innovación y creatividad (Gervilla, 2008) podemos entender la creatividad siguiendo a R. Marín como una innovación valiosa.

Al hablar del ser humano y su desarrollo creativo, si hacemos un recorrido filosófico por la imagen del hombre en el tiempo, es común comenzar por la definición de Aristóteles: "El hombre es el animal racional». Genéricamente se sitúa entre otros animales, pero constitutivamente se define por su racionalidad, que de forma específica lo separa de la bestia. Esta definición de racionalidad parece abocarnos a una visión del ser humano como un ser hecho, terminado, acabado; sin embargo, como señalan Scheler ${ }^{3}$ y muchos otros, el ser humano se halla siempre abierto a "algo", inacabado, inconcluso, indeterminado... al contrario que los animales, que se mueven en un círculo muy estrecho, ajustado - por el instinto- a lo que tiene importancia para su vida y cuya capacidad para aprender, si la poseen, no supera los límites de este marco fijo.

El ser humano ocupa una posición singular en la naturaleza, pues, aunque su dotación física y su deficiencia instintiva le sitúan de forma precaria ante el medio físico, estas deficiencias están ampliamente compensadas por su capacidad de aprender y de modificar el entorno, hasta hacerlo útil para su vida. La condición de "ser que aprende" le permite una gran libertad de acción, ya que su existencia no se ve determinada por la presión innata de los movimientos instintivos, precisos pero a la vez restrictivos. Su capacidad de aprendizaje y la influencia orientadora del medio están incluidas en el plan de desarrollo biológico de la especie, que determina un periodo de inmadurez muy dilatado y una notable retención en el tiempo de los rasgos de la primera infancia.

En palabras de Portman ${ }^{4}$, la cualidad especial del ser humano como ser que aprende guarda relación con su singularidad, con respecto a los otros animales, en el primer año de vida, ya que "en cuanto a la percepción y movimientos, los procesos madurativos operan como situaciones de aprendizaje, bajo la influencia orientadora de su medio a través de la cultura y la educación".

En este proceso de adquisición, desempeña un papel capital la educación, pues el ser humano no puede aprender todo lo que necesita sólo por la experiencia. Esta capacidad de aprender se ve posibilitada por una característica fundamental de la evolución humana, la neotenia, que supone una paulatina ralentización de los ritmos de desarrollo, esto es, un rejuvenecimiento progresivo. Así, hemos evolucionado reteniendo hasta la edad adulta los rasgos juveniles iniciales de nuestros ancestros. Nacemos "como embriones indefensos tras una larga gestación, maduramos tardíamente tras una larga infancia, morimos en edades que tan solo alcanzan los animales de sangre caliente de los tamaños más grandes" (Gould, 1983, 321-322). Permanecemos siempre en estado de desarrollo,

3. SCHELER (1976).

4. Citado por GHelen (1993). 
aunque envejezcamos (Gould, 1983). Somos, básicamente, animales que aprenden; nuestra inmadurez cerebral y la larga infancia permiten el aprendizaje cultural o, dicho de otro modo, la adquisición de modos de conducta por medio de la educación. La educabilidad se encuentra dentro del código genético como posibilidad y como exigencia de que los procesos tengan lugar a través de la mediación social (García Carrasco y García del Dujo, 2001, 121), asimismo, es fruto de los procesos que han tenido lugar durante la evolución. Esta educabilidad del ser humano no es una capacidad estática, sino que se define en términos de proceso; un proceso en el que el grado de educabilidad viene propiciado por la cantidad y por la calidad de los aprendizajes que realiza el sujeto (Castillejo Brull, 1983, 474). Este gran potencial de educación y su indeterminación biológica le abren a la posibilidad de ser creativo y de desarrollar esta potencialidad por medio de la educación.

Creatividad y educación son manifestaciones del desarrollo humano que se entrecruzan, pero que, en sí, son entitativamente distintas. En este sentido, estas páginas tratan precisamente de investigar la relación entre educación y creatividad, para intentar descubrir en qué sentido se complementan o se excluyen mutuamente. Para ello, iniciaremos un breve recorrido por distintos conceptos hasta llegar a unas reflexiones que pretenden atisbar la relación existente entre las mismas. Detectar, en definitiva, la necesidad que para el desarrollo del ser humano en el momento actual tiene la educación para la creatividad.

\section{EduCACIÓN COMO NECESIDAD PARA EL DESARROlLO CREATIVO}

La nota fundamental que diferencia el mundo biológico y físico es, sin duda, la individualidad. La individualidad, nos dice Mayr (1961) tica del mundo orgánico; por ella los individuos, los estadios vitales, los contactos interindividuales, son únicos".

La individualidad humana, como señala Eccles $(1984)^{6}$, se apoya, desde el punto de vista biológico, en la improbable probabilidad ( 1 contra $10^{10.000}$ ) de que vuelva a formarse un cerebro humano idéntico a otro. Pero el desarrollo del individuo depende de la cultura, del contexto humano, de tal forma que, sólo en ese contexto, lo humano logra desarrollarse como tal.

El cerebro humano no madura en el vacío, sino que su potencialidad precisa, para desarrollarse, del contacto cultural. Ahora bien, la entrada en la cultura supone la socialización y, con ella, un cierto abandono de la "singularidad" para adentrarse en lo plural, en los modos de conducta compartidos por un determinado grupo social. Entrar en lo social supone, en cierto sentido, "desindividualizarse", como también entrar en un proceso de adaptación del que depende la supervivencia;

5. MAYR (1961, 1501-1506).

6. Eccles y Zeier (1984). 
asimismo, supone la entrada en el proceso de desarrollo de la posibilidad humana, al no disponer de mecanismos innatos de ajuste al medio. La igualdad biopsíquica humana, no obstante, hace posible que, con independencia de su apariencia física y de su composición genética, pueda vivir y socializarse en cualquier sociedad y pueda aprender cualquier tradición cultural.

El ser humano, frente al animal, puede ser "impresionado" por una multiplicidad de informaciones del mundo exterior, tanto físico como social. Si bien, cuando su supervivencia física está asegurada, es mucho más significativo para él cuanto tiene que ver con lo social. En conexión con esta información, el ser humano "idea", proyecta su acción, valora y elige. Su elección es intencional: su actividad sigue el camino de actuar sobre el mundo con fines propios. Pero, además, su conducta se modifica con los datos de la experiencia, buscando siempre el resultado más útil para su vida, contando con lo que aprende en un proceso creador de "cultura" $y$, a la vez, transformador del medio natural y social.

Los antropólogos están de acuerdo en que el aprendizaje cultural elaborado es exclusivo de la especie humana (Carbonell, 2005) ${ }^{7}$; también coinciden en que la cultura es la razón principal de su adaptabilidad y en que la capacidad cultural compartida por los seres humanos se transmite a través de los procesos de socialización y educación gracias a su enorme plasticidad biológica. La cultura es parte constituyente de toda sociedad, pero la realidad social y la cultural no pueden identificarse. En lo cultural se inscriben las creencias, los mitos, los símbolos, los valores, etc., que van más allá de las formas sociales, de la conducta y de los hechos empíricamente observables.

Desde esta perspectiva, educación y socialización se resuelven desde la óptica del infante como una relación de oposición "natura-cultura", para que éste pueda ir más allá de lo biológico. No obstante, la educación no es mera socialización, es algo más. La socialización tiene como posibilidad ideal que, desde su condición biológica, el ser humano llegue a ser "lo común con los otros" (lo social), por la adquisición de unos determinados "modos de conducta social" que le proporcionan abrigo y seguridad. La educación, sin embargo, se propone una tarea más ambiciosa: tiene como ideal que el educando no sólo llegue a ser lo social, sino a "ser uno mismo"; le abre a la posibilidad más arriesgada de la elección libre, según sus ideales de belleza, justicia, bondad, etc. Le abre a la superior libertad, que supone el distanciamiento de lo biológico y de lo social, para acercase a lo esencial del anthropos. En este punto, la educación puede resolverse no ya como oposición entre educador y educando, sino también como autoeducación. La educación le posibilita "ser uno mismo" y con ello, se implementa la posibilidad de crear.

Es conocido el papel que desempeña la educación en el desarrollo de las llamadas competencias básicas entendidas como conocimientos, capacidades y

7. Carbonell (2005). 
actitudes que permiten a las personas en las sociedades avanzadas vivir, trabajar y adquirir conocimientos. Estas competencias permiten saber hacer y constituyen la base fundamental para el aprendizaje; como, también, es conocido el papel que juega la educación en el desarrollo de toda otra serie de capacidades como son el pensamiento crítico, la creatividad, la capacidad de iniciativa, la resolución de problemas, la evaluación del riesgo, la toma de decisiones y la gestión constructiva de sentimientos y emociones, que intervienen directamente en el comportamiento innovador.

En síntesis, la naturaleza del anthropos nos habla de la necesidad de aprender, de la necesidad de cultura, de la necesidad de educación. Pero también de la posibilidad de la educación, de desarrollar la creatividad.

\section{LA CREATIVIDAD COMO POSIBILIDAD}

El término creatividad, acuñado por Guilford hace más de medio siglo, abarca en la actualidad un gran campo semántico: hablamos de la creatividad del artista, del inventor, del científico, del publicista... El elemento común que subyace a todos ellos en la consideración de creativos es la producción de algo nuevo, distinto. Sin embargo, la novedad no es condición suficiente, ya que si una persona produce algo "nuevo" pero no es bueno, no se puede considerar creativa: lo nuevo ha de ser también valioso. La evaluación como valioso implica un juicio de valor sobre algo, sobre el producto de la acción creadora.

La creatividad es, por tanto, la producción de algo nuevo y valioso (Marín, 1980). Ambas condiciones deben ir unidas para poder calificar una obra de arte, una idea, un producto, la resolución de un problema, un juego o cualquier otro hecho, como creativo.

La creatividad es posible gracias a la indeterminación biológica humana. Por ello, la posibilidad de ser creativo no es patrimonio de unos pocos, crear es universal. El ser humano es por naturaleza creativo, aunque tal posibilidad puede y debe ser cultivada. Crear es someter las operaciones mentales, la inteligencia, a un proyecto creador. Por eso, como afirma E. Faure, en su famoso informe para la UNESCO, las facultades creadoras se encuentran al mismo tiempo entre las más susceptibles y capaces de desarrollo, y las más susceptibles al retroceso e involución. Se aprende a ser creativo o no, y todo lo que se aprende puede enseñarse. Sin embargo, no hay que olvidar que la creatividad es un fenómeno complejo, dinámico y procesual.

Esta postura de universalidad de la creatividad y su necesidad de formación ha sido defendida por los autores más significativos en los estudios sobre la creatividad: Guilford, Torrance, Marín, Allen, Novas, Foster, por citar algunos. En la misma línea J. Nieto (2008) entiende que la innovación se materializa en un proceso que el ser humano realiza de "manera habitual". Innovación de rutina. 
Sin embargo, el ser humano tiene la posibilidad de innovar, de crear alcanzando óptimos de mejora en un proceso intencional que tiene como resultado un producto, una innovación valiosa.

Ello requiere conducir los procesos de aprendizaje hacia el proceso creativo, de innovación de tal forma que puedan integrarse diversos tipos de conocimientos y aunque el conocimiento especializado puede ser muy determinante, el conocimiento tácito sigue siendo vital en el mismo.

Hablamos de creatividad en referencia al producto. Pero también refiriéndonos al proceso; un proceso de formación de ideas, de realización y de verificación de éstas, de comunicación de los resultados finales... Sujeto, producto y proceso son los elementos esenciales del hecho creativo.

Consideramos sujeto, proceso y producto creativos como elementos claves para una visión completa de la creatividad, máxime cuando son aspectos inseparables de la misma. Todos son parte integrante, y a todos hay que atender en el diseño de una estrategia holística y global del hecho creativo. Todos ellos se pueden ver o no favorecidos por el entorno, que, de hecho, puede potenciar, inhibir o incluso cercenar la creatividad.

En referencia al sujeto, la creatividad se define por el pensamiento divergente por la originalidad, la flexibilidad, la independencia, la motivación por el logro, la sensibilidad, la capacidad de inventiva, la imaginación, etc. Por la capacidad de cambiar los "patrones de percepción" establecidos por la experiencia para poder desarrollar "patrones de acción". Lo fundamental es desarrollar actitudes creativas, pero, también, hay que desarrollar otras potencialidades como autorreferencialidad, creer en sí mismo (autoconfianza), proactividad, saber dilatar la gratificación, saber superar la frustración, perseverar en el logro; así como también tener imaginación, curiosidad e interés, sentido crítico, asumir el pensamiento grupal y trabajo en equipo. Estas características de índole personal que son fundamentales en la creatividad están también en la base del espíritu emprendedor de creación de empresas (Castells y Vilaseca, 2007).

En cuanto al proceso se entiende aquí como una herramienta del pensamiento que puede ser empleada deliberadamente para producir un resultado. Entendiendo que el resultado, el producto creativo solo, puede ser valorado, admirado; mientras que el proceso creativo, como cualquier proceso, puede ser practicado, aprendido y enseñado, es decir, puede mejorar con el entrenamiento.

El proceso creativo tiene un carácter interactivo que evoluciona en el tiempo, ya que el ser humano puede absorber, crear e intercambiar conocimiento de forma interdependiente, muchas a veces a través de un proceso continuado de aprendizaje interactivo con el medio, porque la innovación requiere una base amplia y diversa de conocimiento. Precisa tener nivel de inventiva, tener una idea, un proyecto y ser capaz de desarrollarlo. Exige como requisito tener la capacidad de utilizar las ideas, fuera del sistema de juicios, porque el juicio nos mantiene dentro de los canales de la experiencia, lo que imposibilita el proceso de creación. Las ideas han de concretarse, elaborarse, desarrollarse, ponerse a prueba, evaluarse 
y modificarse. También es necesario ser capaz de escapar de la idea dominante al uso, para poder atender la afluencia de nuevas ideas. Precisa de estímulo, de intuición, de orientación y perseverancia en el logro, de incentivar el esfuerzo, porque sólo así es posible, finalmente, superar el medio y su resistencia.

Durante el proceso es imprescindible el pensamiento divergente como también el pensamiento crítico que ante múltiples opciones defina la dirección. Es más, el pensamiento crítico que busca al definir la opción con mayor parsimonia, se constituye, en sí mismo, en un proceso generativo de ideas, pues genera subobjetivos, que pueden permitir incluir requisitos valiosos y con mayor adecuación. El pensamiento crítico contribuye a mejorar el producto creativo. En suma, el pensamiento crítico puede no ser creativo, pero el desarrollo creativo no puede dejar de ser crítico.

Para implementar el proceso creativo pueden utilizarse, según distintos autores (Marín, De Bono, etc.), diversas estrategias, que pueden mejorarlo. Entre las más comunes tenemos el brainstorming (torbellino de ideas), la analogía, transformaciones imaginativas, enumerar atributos, liberarse de la idea dominante, etc. De igual forma, la investigación sobre la creatividad en la solución de problemas parece constatar que los procesos que llevan a soluciones creativas exigen producir un plan de alto nivel antes de intentar la solución del problema. No obstante, la tesis predominante sobre los procesos cognitivos que subyacen a la creatividad es que éstos no se diferencian substancialmente de las otras clases de pensamiento.

En cuanto al producto creativo, la exigencia es que debe materializarse finalmente en algo nuevo y valioso. Se resuelve respondiendo al objetivo propuesto en términos de adecuación. Debe dar respuesta hoy, en un contexto de cambio, a la solución del problema de la vida, el diseño, la ciencia o el arte. Siempre supondrá "formas nuevas de hacer". Estaremos ante una innovación.

Los elementos fundamentales del producto son la originalidad y la adecuación, dado que un producto perfectamente adaptado, que careciese de originalidad, no podría ser considerado creativo, y un producto inadecuado para su contexto, por original que sea, no puede ser considerado creativo. Los productos creativos añaden intuición, inventiva y perspectiva competencial. De igual forma que el criterio sólido, la solución eficaz de los problemas, una percepción aguda, figuran siempre en la confección y acabado de los productos creativos. Los productos creativos "adecuados" producen impacto, rompen moldes y fronteras. Se definen por ser originales y adecuados.

Por otra parte, en la adecuación y la originalidad se precisa tanto del pensamiento creativo (originalidad) como del pensamiento crítico (selección); de hecho, el pensamiento crítico es necesario para que el producto creativo tenga finalmente adecuación. El pensamiento crítico va más allá de la mera selección de opciones, al introducir nuevos requisitos específicos o cualidades finales del producto. Se trata 
de hallar la opción más original, pero que sea igualmente adecuado (Nickerson, Perckins y Smith, 1987, 11) ${ }^{8}$.

En síntesis, los referentes del producto creativo son las cualidades de originalidad, adecuación, impacto, productividad y comunicación.

\section{La CREATIVIDAD COMO UNA DESTREZA ADQUiRIBLE}

La creatividad entendida como una destreza adquirible hace referencia al desarrollo de una competencia que incluye conocimientos, capacidades, habilidades del pensamiento y actitudes creativas. Así, la competencia en creatividad permite al individuo realizar las acciones que conducen a la creatividad de modo óptimo, gracias a las cuales se es capaz de conseguir el logro, la innovación.

No obstante, algunas personas pueden tener especiales aptitudes para la creatividad y estar dotadas para la innovación de forma natural. La competencia creativa tiene, en este sentido, un carácter dado. Sin embargo, aquí nos interesa especialmente la competencia creativa que depende fundamentalmente del aprendizaje y la experiencia de los sujetos. Competencias que, en definitiva, sirven para llevar a cabo una tarea, el "saber hacer" creativo; es decir, competencia vinculada al potencial humano de transformación de la realidad en el sentido en que Chomsky atribuía a la competencia lingüística: "la capacidad de producir infinitamente". Productividad que es, por otra parte, uno de los indicadores de la creatividad.

La creatividad, así, deberá interpretarse no tanto como una cualidad, sino como un modo de actuar que pone el acento en los procesos, en las distintas formas de llegar a diferentes resultados, en el aprender a través de la forma de hacer. Es ésta la clave para que la creatividad no quede en un mero ejercicio imaginativo. Esto obliga a interpretar cada situación, a extraer sus conclusiones y a actuar sin someterse a los códigos preestablecidos, aunque no se tengan garantías de éxito, ni seguridad en el resultado final.

En este sentido, De Bono (1988) entiende que es un error considerar que la creatividad sea una facultad que sólo posee el genio, el inventor o el artista. Su teoría del pensamiento lateral, por el contrario, consiste en pensar que todo el mundo puede aprender a ser creativo, en otras palabras, que puede obtener con su esfuerzo las habilidades necesarias que le permitan tener un comportamiento creativo.

Ser creativo es, para De Bono (1988), una habilidad y una destreza que se adquiere mediante la práctica, una mezcla de actitudes y técnicas. Desde esta perspectiva, la base de la creatividad es hacerse con nuevos procedimientos y nuevas ideas que funcionen; es salir de las rutas trazadas por la experiencia, para poder innovar y crear nuevas formas de acción. Se parte del hecho de que el pensamiento

8. Nickerson, Perckins y Smith (1987). 
es una destreza a potenciar, por lo que "enseñar a pensar" supone conseguir nuevas habilidades para el pensamiento. Los programas diseñados para "enseñar a pensar" ofrecen experiencias por medio de las cuales se modifican, por entrenamiento, las habilidades mentales.

\subsection{Patrones de percepción y desarrollo creativos}

La percepción es la manera en que la mente organiza la información y la dota de sentido creando patrones que automatizan y que dotan de seguridad a la acción. Las estrategias perceptivas se aprenden a fuerza de observar, identificar y obtener regularidades. La información que recogemos a través de estas estrategias perceptivas nos permite establecer relaciones entre las cosas, ampliar o modificar nuestra concepción de la realidad. Mediante la experiencia, se construyen modelos de percepción (acerca de cómo son las cosas) y los consiguientes modelos de acción (cómo actuar). Se extraen patrones fijos que introducen orden y sentido en el medio, a través de ellos se obtienen las legalidades: se percibe el mundo. En definitiva, estos modelos se automatizan por la experiencia.

La mente opera así mediante la creación de modelos que necesitan ser revisados, renovados constantemente, para adaptarse a un medio ambiente cambiante. A veces, el cambio es tan grande que la revisión no es suficiente, sino que es preciso innovar, crear nuevas formas de percepción. "Cambiar las percepciones, mirar de nuevo, para poder aportar soluciones nuevas, creativas, adaptadas al cambio" (De Bono, 1988). De todo ello se deduce que el primer estadio del pensamiento creativo es el de la percepción; es éste un paso fundamental en el desarrollo creativo, ya que un cambio en esta primera fase del pensamiento es necesario para que pueda emerger la creatividad. El segundo estadio del pensamiento creativo consiste en generar ideas nuevas. Pero esta segunda etapa sólo comienza si se ha desarrollado bien el estadio perceptivo, es decir, si la flexibilidad ha permitido nuevas formas de ver las cosas. El objetivo aquí es hacerse con nuevos procedimientos e ideas que funcionen. Se trata de posibilitar el cambio de las percepciones cambiando la forma en que miramos las cosas. Sólo así es posible tener nuevos y mejores patrones de acción y, finalmente, la producción de algo nuevo y valioso. El requisito fundamental en este punto es tratar los datos creativamente.

La creatividad, en definitiva, se convierte, así, en la capacidad de revisar nuestros patrones de percepción y cuestionar nuestras formas de pensar o actuar, de romper con los itinerarios lógicos para valorar la necesidad de cambio y de ampliación de nuestra realidad.

Hoy en día lo fundamental no son los contenidos, puesto que en cualquier ordenador o base de datos están. Se necesitan destrezas y habilidades para dar respuestas creativas (nuevas y valiosas) a los cambios rápidos del mundo. El problema es alcanzar la solución a los problemas que se nos presentan. En definitiva, enseñar a pensar es enseñar a ser creativos, enseñar a generar ideas nuevas para una situación dada. El objetivo es que los individuos vean el pensamiento como 
una habilidad adiestrable y desarrollen la capacidad de incidir sobre su pensamiento hasta formarse como un pensador habilidoso que conoce qué instrumentos utiliza y cómo hacerlo.

En síntesis, el pensamiento lateral que propugna De Bono (1988) tiene como objetivo el cambio de modelos y el cambio en la estructura de esos modelos. En lugar de basar su acción en la combinación, como hace el pensamiento lógico, el pensamiento lateral trata de descomponer las estructuras de los modelos, con el fin de que las partes de éstos se ordenen de forma distinta, entendiendo que la percepción de la información tiene normalmente una influencia decisiva para la acción posterior. Sólo intentando encontrar alternativas que aporten nuevas soluciones se puede llevar a término el proceso creador. Por ello, en esta fase es tan importante ser flexible y original, así como tener fluidez de ideas.

\subsection{La actitud creativa}

La creatividad es la posibilidad de salirse de la repetición y de la rutina neutral para innovar. De esta manera, se puede transformar la forma de enfrentarse con la realidad cambiando el tipo de respuesta que se da, teniendo una actitud creativa. De hecho, numerosas investigaciones han hallado asociaciones constantes entre la creatividad y las actitudes (Nickerson, Perckins y Smith, 1987, 177).

La actitud creativa nos coloca en una multiplicidad de perspectivas, en la posibilidad de "ver de nuevo", en la posibilidad de cambio. La actitud creativa se opone a la inercia, a la rutina de la repetición, a la continuidad, a la misma forma de entender causas y efectos. La actitud creativa precisa salir de los límites estrechos, ir más allá, dejar en suspensión el discurrir automático para poder apreciar otro tipo de relaciones; permite jugar con la metáfora en la descripción de lo que existe, revisar los esquemas que utilizamos para entender la realidad: abrir la puerta a la aparición de respuestas imaginadas.

De todo ello se deduce la importancia de actitudes creativas en el sujeto para implementar la creatividad y la innovación. Para lo cual el primer paso es incidir a nivel cognitivo para desarrollar nuevas creencias, opiniones, en definitiva, patrones de percepción nuevos que permitan librarse de los juicios previos (prejuicios), de las ideas dominantes, etc.; puesto que las creencias, que son esquemas conceptuales que recogen la información que tiene el sujeto acerca de algo, con los suficientes refuerzos, generan actitudes y la actitud precede a la acción.

Las creencias son estructuras cognitivas regularmente estables, pero que el individuo modifica y estructura cuando recibe información que hace cuestionar las mismas. De ahí que cualquier programa de cambio de actitudes debe partir no sólo del conocimiento de las creencias del individuo, sino también del conjunto de variables externas o circunstancias que determinan que se mantengan dichas creencias. De hecho, existen diferentes técnicas estratégicas que pueden modificar o mejorar las creencias en sentido creativo (comunicación persuasiva, orientada al cambio de pensamiento, modelado, experiencia vivida, etc.). 
Por otra parte, hay que tener en cuenta, en relación a la actitud, sus tres componentes fundamentales (cognitivo, afectivo y comportamental), entendiendo que estos tres componentes no necesariamente se presentan unidos. La gente no siempre se comporta como piensa o siente; por ello, para programar cualquier cambio de actitudes se deben tener en cuenta las percepciones que el sujeto tiene del contexto y actuar sobre esa base para garantizar que finalmente se lleva a cabo la acción (Fishbein y Adjen, 1980).

Lo que caracteriza a la actitud es la evaluación afectiva de los resultados positivos o negativos de la realización de una determinada conducta. Por ello hay una diferencia esencial entre creencias y actitudes, ya que mientras las primeras hacen referencia directa al ámbito cognitivo, las segundas hacen referencia al ámbito de lo afectivo.

Por ello hay que conseguir que el sujeto considere valioso colaborar en proyectos o actividades que promuevan el ver de nuevo, la creatividad, la innovación. No obstante, aunque tener determinadas creencias, apreciar el valor de la creatividad, permite generar actitudes positivas hacia el cambio y la innovación, ello no garantiza que el individuo se comporte necesariamente de acuerdo con las mismas. Para garantizar que finalmente actúe y se comprometa con el desarrollo creativo debe esperar algún tipo de resultado positivo de su acción, que le sirva de incentivo y refuerzo, ya sea desde la autorreferencialidad, por el reconocimiento externo profesional o social, o desde la satisfacción de beneficiar a alguien, etc. Por ello, la incentivación por el logro se convierte en un buen camino para desarrollar actitudes creativas. Las técnicas organizacionales (teoría "Z", círculos de calidad, etc.), que promueven la delegación de poder, promueven la participación e incentivan las buenas ideas, se muestran idóneas para conseguir una mayor afluencia de ideas, procesos y productos creativos.

Esta forma de desarrollo creativo se puede y se debe potenciar mediante la educación, desarrollando creencias y actitudes adecuadas, entendida como procedimiento que permite implementar nuevas ideas y procesos creativos en cualquier orden de la vida, porque sólo así es posible preparar a las nuevas generaciones para un mundo en constante cambio.

\section{Creatividad y educación}

La educación para la creatividad ha de atender, desde los primeros años, al desarrollo de la "capacidad de pensar" desde la imaginación. La infancia es la etapa de buscar, de descubrir, preguntar y aprender. Por ello, se ha de contar con un clima motivador y la debida incentivación del comportamiento innovador, para conseguir que el potencial creativo humano se desarrolle desde la infancia y durante toda la vida. Los niños, por naturaleza, están interesados en conocer, y disponen, espontáneamente, de diversos modos de investigar. Esto incluye imaginar, fantasear, preguntar, adivinar, experimentar y extrapolar tendencias. Para enseñar a los niños creatividad, debemos conocer estas tendencias naturales, partir 
de ellas, reforzarlas y convertirlas en poderosos instrumentos para investigar sobre el entorno y cambiarlo hacia direcciones deseables.

Sin embargo, numerosas investigaciones señalan que la creatividad de los niños decrece con los años de permanencia en el sistema educativo9, de forma que la curiosidad, la búsqueda creativa, da paso con el tiempo a comportamientos más rígidos, convergentes e inflexibles. Aunque no podemos entrar aquí en la determinación de las causas, sí queremos señalar algunos de los factores inhibidores de la creatividad en el aula.

\subsection{Factores inbibidores de la creatividad}

- Los esquemas de percepción y de acción enseñados a los niños en los primeros años se presentan en general con un carácter convergente, unívoco, como los únicos válidos, sin dar oportunidad a otras formas de percepción ni a otros esquemas de acción.

- La motivación por el logro se prima constantemente en la familia y en la escuela. Las buenas calificaciones y la acomodación a los demás constituyen el éxito visible y públicamente reconocido. Se trata de una motivación extrínseca, de adaptación a los parámetros y valores evaluativos del grupo. Por el contrario, para fomentar y no inhibir la creatividad, la motivación (generalmente externa) deberá invertirse y basarse prioritariamente en el placer de descubrir, inventar, seguir nuevos caminos... En esta motivación deseable (predominantemente intrínseca) deben primar las estrategias, los caminos divergentes hacia la solución, más que el logro convergente y esperado.

- La búsqueda de la propia identificación con el grupo lleva muchas veces a los niños a desarrollar comportamientos idénticos a los otros, así evitan desarrollar conductas y rasgos propios que podrían considerarse "distintos", simplemente para no hacer el ridículo, sin dar posibilidad, de esta forma, a la autonomía y la creatividad.

- La actitud interrogativa natural del $2 .^{\circ}$ al $5 .^{\circ}$ año de vida, que posibilita la creatividad, puede bloquearse $-\mathrm{O}$ hasta suprimirse- si no se priman y se estimulan sus preguntas, incluso de índole crítica. La prohibición expresa o el miedo al ridículo impiden arriesgarse y aprender a preguntarse correctamente, bloquean la creatividad. No hay que olvidar que la auténtica interrogación surge en la infancia de la espontaneidad, de la curiosidad y de la necesidad de aprender.

9. Pérez Alonso-Geta (1981). 
- Finalmente, las presiones de distinta indole que pueden ejercerse en la familia y en la escuela actúan como potentes factores que inhiben la creatividad.

\subsection{Hacia una educación creativa. El desarrollo de espacios creativos}

Para Torrance es evidente que a los niños se les debe y se les puede enseñar creatividad desde los primeros años, de tal manera que sus habilidades de pensamiento creativo y los hábitos innovadores que adquieran sean útiles para la solución de problemas e incluso para adquirir las tradicionales destrezas educativas. Estas habilidades, sin duda, no son medibles por los test tradicionales de inteligencia y de aptitudes escolares, pero son importantes para el desarrollo personal y profesional. Por ello, la educación en la creatividad y la innovación debe potenciarse en el contexto escolar, para evitar los factores que inhiben la creatividad.

De igual forma, cualquier proyecto educativo que, de forma global, pretenda desarrollar la creatividad debe atender a los tres elementos claves del hecho creativo: sujeto, proceso y producto. Si bien ha de centrarse, en primer lugar, en la formación del sujeto: debe desarrollar su flexibilidad, a fin de poder cambiar los patrones de percepción que permiten ver de nuevo. Para ello, es necesario generar creencias favorables al cambio y la innovación que permitan desarrollar actitudes positivas hacia la creatividad, ya que la actitud precede a la acción.

Del mismo modo, también es necesario desarrollar la seguridad en sí mismos, que les permita arriesgarse y perder el miedo al fracaso, ayudarles a superar la frustración y los obstáculos, sin paralizarse ni abandonar o derivar la frustración en agresividad, sino mediante el desarrollo de nuevas estrategias. Pensar de nuevo exige abandonar los caminos automatizados, y dilatar la respuesta, así como dilatar la gratificación. Sólo así el sujeto podrá involucrarse de lleno en el proceso creativo; un proceso que exige tanto generar ideas nuevas, como esfuerzo para superar el medio y su resistencia. Sólo así se puede obtener el producto creativo, que será algo nuevo y valioso: un producto innovador.

Pero además es necesario "promover la creación de nuevos escenarios, nuevos espacios antropológicos desde los que se facilite la innovación, la creatividad". Estos espacios deben permitir utilizar el azar, error e incluso la frustración como momentos claves para deshacerse de las ideas dominantes y promover patrones de percepción, nuevas ideas y procedimientos.

De hecho, sabemos que los factores socioambientales, aunque no son consustanciales a la creatividad, sí pueden determinar el carácter creativo o no de las tareas. Pueden fomentar o inhibir el pensamiento creativo y la motivación intrínseca o, lo que finalmente es lo mismo: las actividades creadoras.

Los espacios de socialización y educación pueden, de hecho, inhibir o fomentar la creatividad, o hasta hacer de ella, en sentido positivo, una cualidad definitoria de la formación de determinados grupos humanos. 


\subsubsection{El espacio creativo}

El espacio físico, a lo largo de la historia del pensamiento, se ha considerado desde dos vertientes diferentes: como espacio "continente" o receptáculo de todos los cuerpos y como espacio "relación". Esta idea de espacio como relación está en la base del espacio antropológico; hace referencia a las relaciones que los individuos en ese entorno construyen.

Certeau define el espacio social como un lugar "practicado". Son los caminantes los que transforman la calle, geométricamente definida por el urbanismo como lugar, en un espacio social. También Merleau Ponty en su Fenomenología de la percepción distingue "el espacio geométrico" y el "espacio antropológico". Este último sería un espacio "existencial", lugar de una experiencia de relación con el mundo.

El concepto de interacción individuo-espacio hace referencia a un proceso de intercambio mutuo; por una parte, el medio sirviendo de entorno; de otra, el sujeto que introduce cambios en su medio. El encuentro activo entorno-sujeto daría lugar a la creación del espacio-propio, del "espacio de sí mismo", a través de un acto de cocreación personal. Su cualidad personal como ser humano depende directamente del alcance de su participación creadora en los distintos ámbitos, de su capacidad de generar sentido, de establecer lazos de presencia, colaboración y compromiso con las realidades del entorno, de su capacidad de crear espacios propios. A través de su actividad cocreadora va construyendo "su mundo", a base de elegir en el vasto y complejo campo de posibilidades que le ofertan. Es éste "un mundo personal" constituido por él dentro de unas coordenadas espacio-temporales globales. La expresión "mundo propio" remite, como vemos, a la idea de posibilidad creadora, de originalidad, de autonomía de innovación y creatividad.

Para Auge (1994), el lugar se definirá como lugar de "identidad", "de relación". Simboliza la relación de cada uno de sus ocupantes consigo mismo, con los demás y con su historia común. Contrariamente, un espacio en que ni la identidad, ni la relación, ni la historia están simbolizadas para él, será un "no lugar". De otro modo, mientras un lugar es un espacio simbolizado con sus puntos de referencia, sus monumentos y su fuerza de evocación, los "no lugares" son los espacios más despersonalizados. Son aquellos en que las personas coexisten, sin crear espacios propios de relación. En el campo educativo que nos ocupa, crear espacios propios es fundamental para que pueda emerger la creatividad.

\subsubsection{Pilares básicos del espacio creativo en el aula}

- Espacio físico creativo. Creado a través de la organización y gestión del espacio y los recursos. Para ello es necesario crear una atmósfera estimulante, un marco de trabajo lo suficientemente estudiado para permitir a los niños construir su espacio. Hacer que éste se convierta en un "lugar" donde puedan expresarse en una diversidad de formas. La organización escolar deberá contemplar y acomodar una diversidad de actividades. 
Los profesores deben disfrutar de su papel al establecer el escenario en el que los niños aprendan a expresarse en una diversidad de formas.

- Espacio intelectual creativo. Estimulado a través del fomento de la imaginación y de potenciar el "ver de nuevo", mediante la creación de nuevos patrones de percepción y de acción. Para ello es preciso potenciar la capacidad imaginativa de los niños, ya que desempeña un papel fundamental en el desarrollo de la comprensión de la realidad y la capacidad de simular nuevas realidades, acciones y mundos posibles sin experimentarlos en realidad. La imaginación ha de desarrollarse para permitir superar las imágenes restringidas de una imaginación que nunca o pobremente ha sido nutrida. Por ello se precisa flexibilizar la percepción infantil, mediante la generación de nuevos patrones de percepción de la realidad que permitan nuevos patrones de acción, para adecuarse mejor al cambio. El desarrollo de sus capacidades y potencial intelectual a través de la imaginación y del pensamiento y expresión creativos.

- Espacio socioemocional creativo. En el que se potencien las competencias socioemocionales infantiles básicas que permiten tener confianza en uno mismo, disfrutar de la relación con los demás o comprender y canalizar adecuadamente sentimientos y emociones. Para ello, es fundamental potenciar en los niños la capacidad de entender y comprender emociones y sentimientos propios y ajenos. Desarrollar una autoestima positiva y realista que les permita sentirse seguros, atreverse a correr riesgos, a experimentar sin miedo al fracaso y a percibir las sensaciones que produce el éxito. Potenciar la capacidad de gestionar y controlar impulsos, sentimientos y emociones, dejando al sujeto libre para experimentar y crear.

\section{A MODO DE CONCLUSIÓN}

La formación del ser humano hoy, en un contexto de incertidumbre y profundos cambios sociales y tecnológicos, es sin duda incompleta si no se desarrolla la creatividad. Sin embargo, a pesar de la difusión que "la creatividad" en nuestra sociedad, como concepto científico y objeto de estudio, ha tenido, su desarrollo todavía es más una situación deseable que una realidad. En la actualidad la creatividad no deja de ser para algunos un término más o menos de moda en la búsqueda de la originalidad disciplinar de determinados expertos.

De hecho, como señala Alonso (2000) la creatividad se percibe socialmente con una ambigüedad tal que de hecho la ha condenado al ostracismo. En términos generales, se es resistente a la misma. Efectivamente, las estructuras sociales e institucionales (también la escuela) están basadas en la conservación más que en el descubrimiento. En la estructura social priman los cauces establecidos, los caminos recorridos, los patrones de percepción y de acción consolidados. Sin embargo, frente a esta imagen funcional y eficiente socialmente aceptada, prevalece como 
en la vida lo impredecible, lo variable y lo ambiguo. No hay garantía, por ejemplo, de que los objetivos formativos que se proponen sirvan en la sociedad del futuro. Tal incertidumbre hace del espacio social un lugar donde la creatividad aparece, aunque sea tan sólo como posibilidad. Por eso, es sin duda imprescindible desarrollar su potencial.

En términos generales, la educación de la creatividad se promueve al dinamizar las potencialidades individuales: favoreciendo la flexibilidad mental, la originalidad, la inventiva, la receptividad de las nuevas ideas y la autonomía. Todo ello junto con la competencia emocional y social. Para ello, es necesario que los educadores proporcionen un ambiente de libertad, técnicas y medios precisos, de modo que se posibilite el desarrollo de la capacidad creadora en un ambiente de libertad.

El desarrollo de las estrategias creativas, el cambio de patrones, enseñar a pensar, deben considerarse, desde los primeros años, como un elemento esencial en el proceso educativo. Es necesario enseñar y ejercitar a los niños para consolidar los elementos básicos de su actitud creativa hacia el entorno y hacia el mismo proceso de aprendizaje. El desarrollo de estas estrategias exige crear espacios creativos, a través de la organización y gestión del espacio y los recursos; a través del fomento de la imaginación (el "ver de nuevo") y de la capacidad de descubrir y buscar nuevas respuestas y pautas de acción.

En suma, es necesario estimular desde la escuela la creatividad, que se cimienta sobre soportes básicos como la flexibilidad, la autonomía y libertad, la autoestima y la actitud positiva ante la vida y el cambio: cualidades todas ellas que permiten afrontar la realidad desde formas y enfoques diferentes, ya que, no en vano, el pensamiento creativo se erige en momentos de incertidumbre como un factor dinamizador de la personalidad integral y de la misma sociedad. Se necesita de la educación.

\section{BibliografíA}

Acosta, M. (1998) Creatividad, motivación y rendimiento académico. Málaga, Aljibe.

Allen, S. (1963) Creatividad morfológica. México, Herrero Hermanos.

Alonso, C. (2000) Qué es la creatividad. Madrid, Biblioteca Nueva.

Auge, M. (1996) Los "no lugares". Una antropología de la sobremodernidad. Barcelona, Gedisa.

BeAn, R. (1993) Cómo desarrollar la creatividad en los niños. Madrid, Debate.

Bонм, D. (1993) Sobre la creatividad. Barcelona, Kairos.

CARBOnell, E. (coord.) (2005) Hominidos: las primeras ocupaciones de los continentes. Barcelona, Ariel.

Castells, M. y Vilaseca, J. (2007) Entorno innovador, iniciativa emprendedora y desarrollo local. Barcelona, Octaedro.

Castillejo, J. L. (1981) Nuevas perspectivas de las ciencias de la educación. Madrid, Anaya.

- (1983) Educabilidad, en Diccionario de Ciencias de la Educación. Barcelona, Diagonal.

Claxton, G. (1999) Cerebro de liebre, mente de tortuga. Barcelona, Urano. 
DabDoub, L. (2008) Desarrollo de la creatividad. México, Editorial Esfinge.

De Bono, E. (1988) El pensamiento lateral. Barcelona, Paidós.

- (1997) Seis sombreros para pensar. Barcelona, Editorial Granica.

De lA TORRe, S. (1987) Educar en la Creatividad. Madrid, Narcea.

- (1997) Innovación educativa. Madrid, Dikinson.

ECCles, J. C. y ZeIER, H. (1984) El cerebro y la mente. Barcelona, Herder.

Fishbein, M. y Adjen, I. (1980) Understanding attitude and Predicting social behavior. New Jersey, Prentice Hall.

Foster, J. M. (1971) Creativity and the teacher. London, Macmillan.

García Carrasco, J. y García Del Dujo, A. (2001) Teoría de la Educación II. Salamanca, Ediciones de la Universidad de Salamanca.

Gehlen, A. (1993) Antropología filosófica. Barcelona, Paidós.

Gervilla, A. (2008) Creatividad, Calidad e Innovación. Curso de doctorado UCM en Line research https://www. Search. m.s.m.com. Consultado en noviembre 2008.

Goleman, D.; Kaufman, P. y Ray, M. (2000) El espiritu creativo. Buenos Aires, Ediciones B.

Gould, S. J. (1983a) Desde Darwin: reflexiones sobre historia natural. Madrid, Hermann Blume.

- (1983b) El pulgar del Panda. Madrid, Hermann Blume.

GuILFORD, J. P. (1968) Intelligence, creativity and their educational implications. San Diego, Kirapp.

- (1971) The structure of intellect. New York, McGraw Hill.

- (1977) La naturaleza de la inteligencia humana. Buenos Aires, Paidós.

- (1978) Creatividad y educación. Buenos Aires, Paidós.

López, A. (2003) La innovación de las personas. Madrid, New Jersey, Prentice Hall.

Lowenfeld, V. y LAmbert BritTAin, W. (1984) Desarrollo de la capacidad creadora. Buenos Aires, Kapelusz.

Marín, R. (1980) La Creatividad. Barcelona, CEAC.

Marín, R. y De la Torre, S. (1991) Manual de creatividad. Barcelona, Vicens-Vives.

Marín, R.; LÓpez-BARAjas, E. y MARTín, M. ${ }^{a}$ T. (coords.) (1998) Creatividad polivalente. Madrid, UNED.

MATEO, E. y otros (1983) ¿Cómo fomentar la creatividad en la familia y en la escuela? Madrid, Marsiega.

MAYR, E. (1961) Cause and effect in biology: Kinds of causes, predictability, and teleology are viewed by a practicing biologist, Science, 134, 1501-1506.

Motos, T. (1999) Creatividad dramática. Santiago de Compostela, Universidad de Santiago.

Nickerson, R.; Perckins, D. y Smith, E. (1987) Enseñar a pensar. Barcelona, Paidós.

NIETo, J. (2008) ¿Innovas o abdicas? Valencia, Editorial UPV.

Novas, M. H. (1971) Psicología de la aptitud creadora. Buenos Aires, Kapelusz.

PÉREZ Alonso-GetA, P. M. ${ }^{a}$ (1981) Aptitudes de la producción divergente en EGB, Innovación creadora, 12.

- (1984) Panorámica de los tests de creatividad, Revista de Psicología y Pedagogía Aplicada, vol. 15, 28.

- (2008) Juego, Creatividad y Familia, en Psicología y Gestión Familia. Valencia, PUV.

- (2009) Educación y creatividad. Valencia, PUV (en prensa).

PRADO, D. (1986) Torbellino de ideas, un método universal y participativo innovador e inventivo. Santiago de Compostela, Universidad de Santiago.

Runco, M. (2003) Education for creative potencial, Scandinavian Journal of Educational Research, vol. 47, 3. 
SCHeler, M. (1976) El puesto del hombre en el cosmos. Barcelona, Alba.

Torrance, E. P. (1969) Orientación del talento creativo. Buenos Aires, Troquel.

- (1970) Encouranging creativity in the classroom. Iowa, W. L. Brown.

- (1977) Educación y capacidad creativa. Madrid, Marova.

Torrance, E. P. y MYers, R. E. (1986) La enseñanza creativa. Madrid, Santillana.

VÁzQuez, A. (2008) Estrategias de imaginación. Barcelona, Granica.

WeIsBerg RoBert, W. (1987) Creatividad: el genio y otros mitos. Barcelona, Labor. 